\title{
Developing LHCb Grid Software: Experiences and Advances
}

\author{
Ian Stokes-Rees \\ University of Oxford \\ Department of Particle Physics \\ 2 September 2004
}


* LHCb Particle Physics Experiment developed a computational grid infrastructure, starting in 2002

* Deployed on 20 "normal", and 30 "LCG" sites

* Effectively saturated LCG and all available computing resources during 2004 Data Challenge

* Supported 3500 simultaneous jobs across 50 sites

* Produced, transferred, and replicated 58 TB of data, plus meta-data

* Consumed over 400 CPU years during last 3 months

* Achieved by

$>$ lightweight Services and Agents

$>$ developed in Python

$>$ with XML-RPC interfaces

$>$ and, of course, a lot of blood, sweat, and tears 


\section{Overview}

* Requirements

* Architecture

* Integration with LCG

* Project Management $\quad$ Krasit

* OGSI/GT3 Flop

* Instant Messaging

* Future

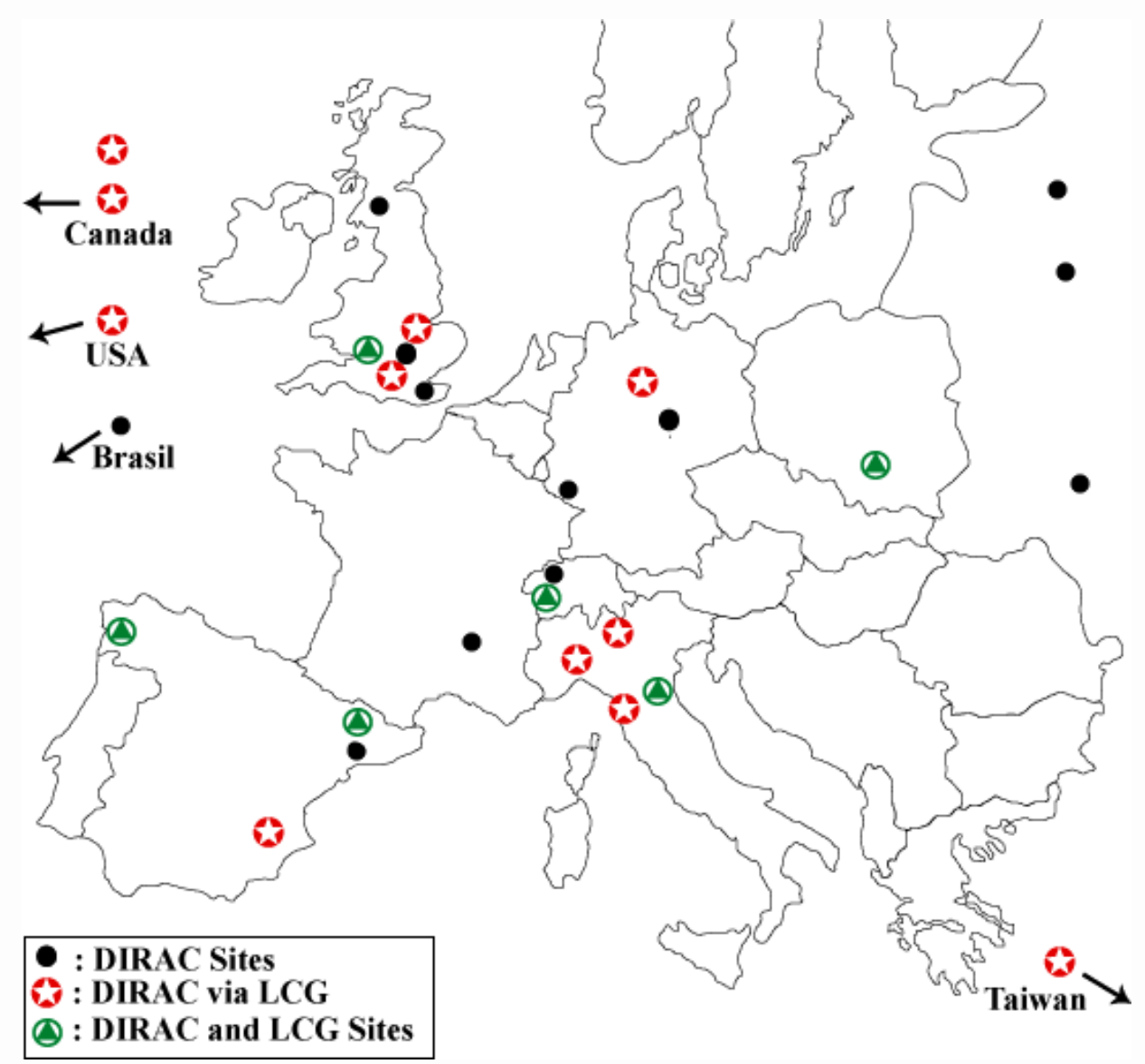

DIRAC Agent Network, July 2004 
* LHCb experiment

$>$ particle physics detector at CERN

$>$ will generate data at $40 \mathrm{MB} / \mathrm{s}$ from 2007

- that's 3.4 TB/day

$>500$ physicists

$>100$ institutes/sites

$>$ simulations already running

$>$ software development and testing underway

Developing LHCb Grid Software UK e-Science AHM, Sept 2004

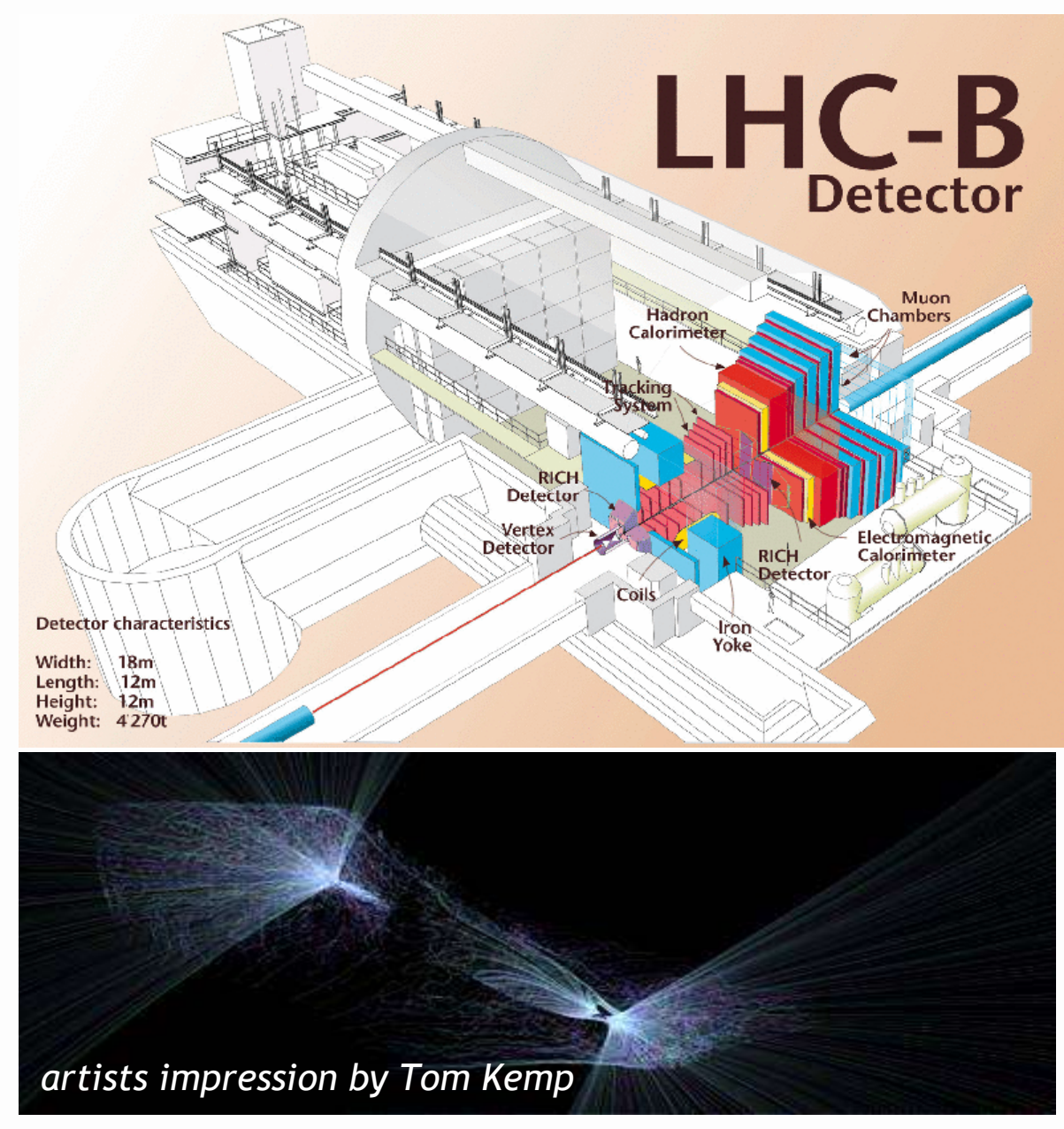


* Required simple integration with existing computing centres

$>$ support different batch systems

$>$ easy for LHCb site representatives to install, configure, and run "Grid Software"

$>$ little or no intervention while running

* Needed to support LHCb computing

$>$ Existing data management and simulation software and services

$>$ Regular software updates

> Large data files with associated meta-data and replication 


\section{0,000 queued jobs}

10,000 running jobs 100 sites

\section{We think this is what computational grids look like}


- But why not just use EDG or LCG? CABin ILCG $>$ In 2002, EDG was not ready for serious use $>$ Lots of existing computing resources still not (yet) tied in to LCG

$>$ LHCb sought to develop a stepping stone to LCG computing

*... and LHCb had some ideas on how to do "grid computing" a bit differently ... 
DIRAC: Distributed Infrastructure with Remote Agent Control

$>$ Service Oriented Architecture

- Services exposed via simple XML-RPC interface

- accessible over HTTP

$>99 \%$ Python pouptom

$>$ DIRAC Agents deployed at computing centres

$>$ Job Pull Paradigm, similar to Condor

- in fact, using Condor ClassAds, and Condor Matchmaker 
Services Agents Clients

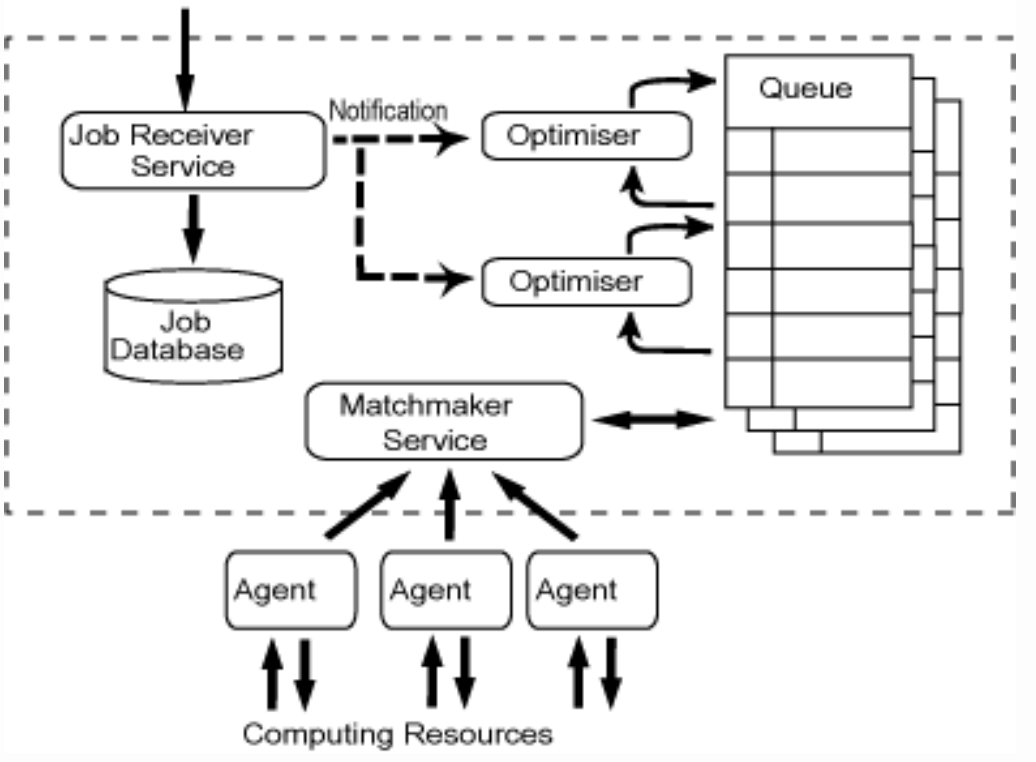

Developing LHCb Grid Software UK e-Science AHM, Sept 2004
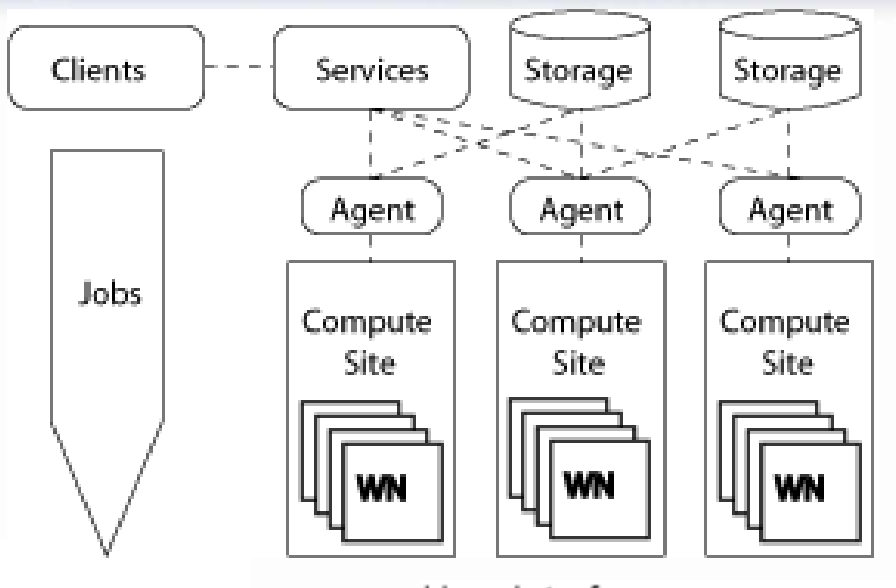

User Interface

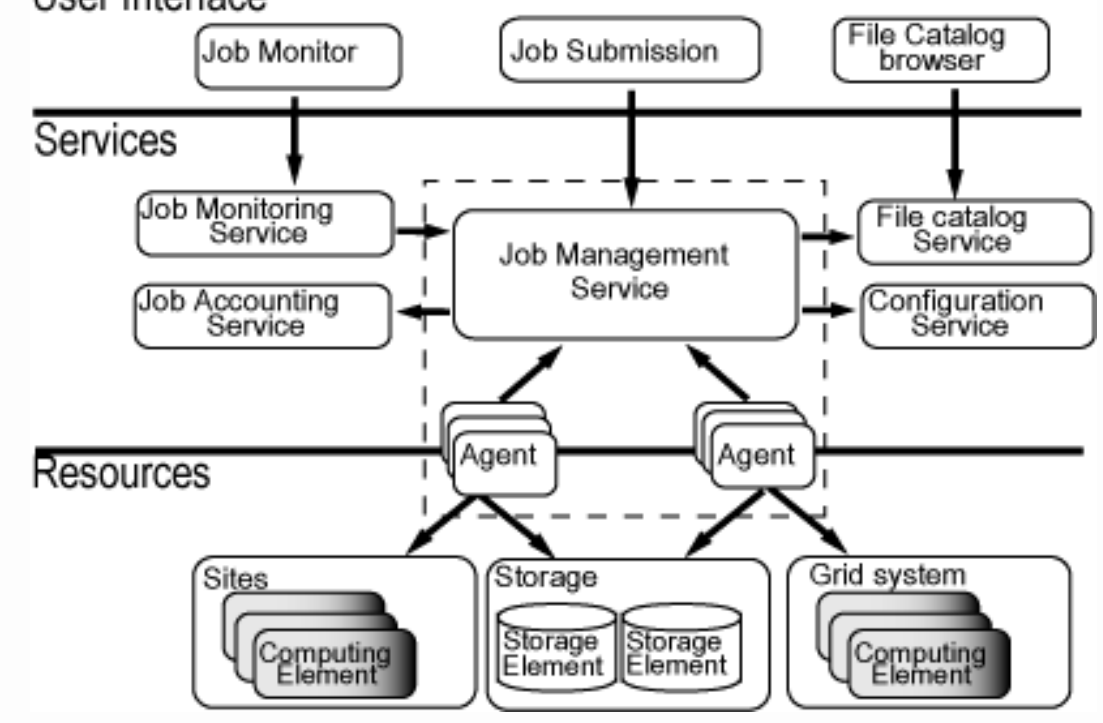

\section{Users Jobs Data}


* DIRAC architecture followed:

$>$ OGSA/OGSI direction towards "grid services"

$>$ Direction of ARDA proposal to EGEE

- Now implemented as gLite

* DIRAC was meant to fit into this brave new world of Grid Services

$>$... and we tried (GT3, OGSI, pyGridWare,

Clarens) 
* Dream was that ARDA, possible successor to EDG architecture, would propose a service decomposition and simple, clear, interfaces

$>$ Allow alternative/pluggable/replaceable service implementations

- For competition

- For bug fixing

- For different feature/performance emphasis

$>$ Allow extension of "Grid Functionality" through new services

$>$ Allow rapid development of services 


\section{EnidPp}

Architectural Aspects of DIRAC 
* LHCb Experiment Standardized on Python wherever possible

* I had serious doubts about the performance of an interpreted language for a production grid system

$>$ Proved wrong! Python worked just fine.

* Facilitated rapid development and bug fixing

* Good object oriented construction

* "Dynamic Typing" (aka not type safe) is a challenge and requires careful coding

* "Batteries Included" meant that DIRAC Agents and Clients were super lightweight and only required:

$>1.2$ meg tarball (Python code and associated libraries)

$>$ Python 2.2 interpreter installed

> Outbound internet connection 
* Allowed reconfiguration of overall system

* Encouraged rapid development

* Automatic paralellism

* Easy deployment and maintenance

* Forced separation of functionality

* Scaled well

* Significant complexity of co-ordinating configuration and location of services 


\section{Multi-threaded XML-RPC}

\section{* Fast}

$>40$ queries per second

\section{* Easy}

$>3$ lines of Python

server $=$ ThreadingXMLRPCServer $(\ldots)$

server.register_instance (service)

server.serve_forever()

* Didn't need anything more complicated

$>$ SOAP, WSDL, etc. 


\section{EridPP}

POST /RPC2 HTTP/1.0

User-Agent: Frontier/5.1.2 (WinNT)

Host: betty.userland.com

Content-Type: text/xml

Content-length: 181

<methodCall>

$<$ methodName>examples.getStateName $</$ methodName $>$

$<$ params $>$

$<$ param>

$<$ value $><$ i $4>41</$ i $4></$ value $>$

$</$ param $>$

$</$ params $>$

$</$ methodCall $>$

Developing LHCb Grid Software

UK e-Science AHM, Sept 2004 


\section{EridPP}

POST /InStock HTTP/1.1

Host: betty.userland.com

Content-Type: application/soap+xml; charset=utf-8

Content-Length: nnn

<soap: Envelope

xmlns : soap="http: //www.w3 .org/2001/12/soap-envelope"

soap: encodingStyle="http://www.w3.org/2001/12/soap-encoding">

<soap:Body xmlns:m="http://userland.com/examples">

$<\mathrm{m}:$ GetStateName>

$<\mathrm{m}:$ Index $>41</ \mathrm{m}$ : Index $>$

$</$ m: GetStateName $>$

$</$ soap: Body $>$

$</$ soap: Envelope $>$

Developing LHCb Grid Software

UK e-Science AHM, Sept 2004 
* Unreasonable to ever expect a single machine to schedule all jobs in the grid, or even all jobs for a VO

$>$ Assumes complete view of system

$>$ Assumes up to date information

* Push scheduling introduces single point of failure and overloading in presence of 1000s of jobs (NP hard)

* Pull scheduling is Obviously Better

$>$ Resources ask for jobs when they are ready

$>$ Job serves "next best job" for that resource 
* Averaged 420ms match time over 60,000 jobs

$>$ Using Condor ClassAds and Matchmaker

* Queued jobs grouped by categories

- Matches performed by category

- Typically 1,000 to 20,000 jobs queued

* We still suffered from single point of failure

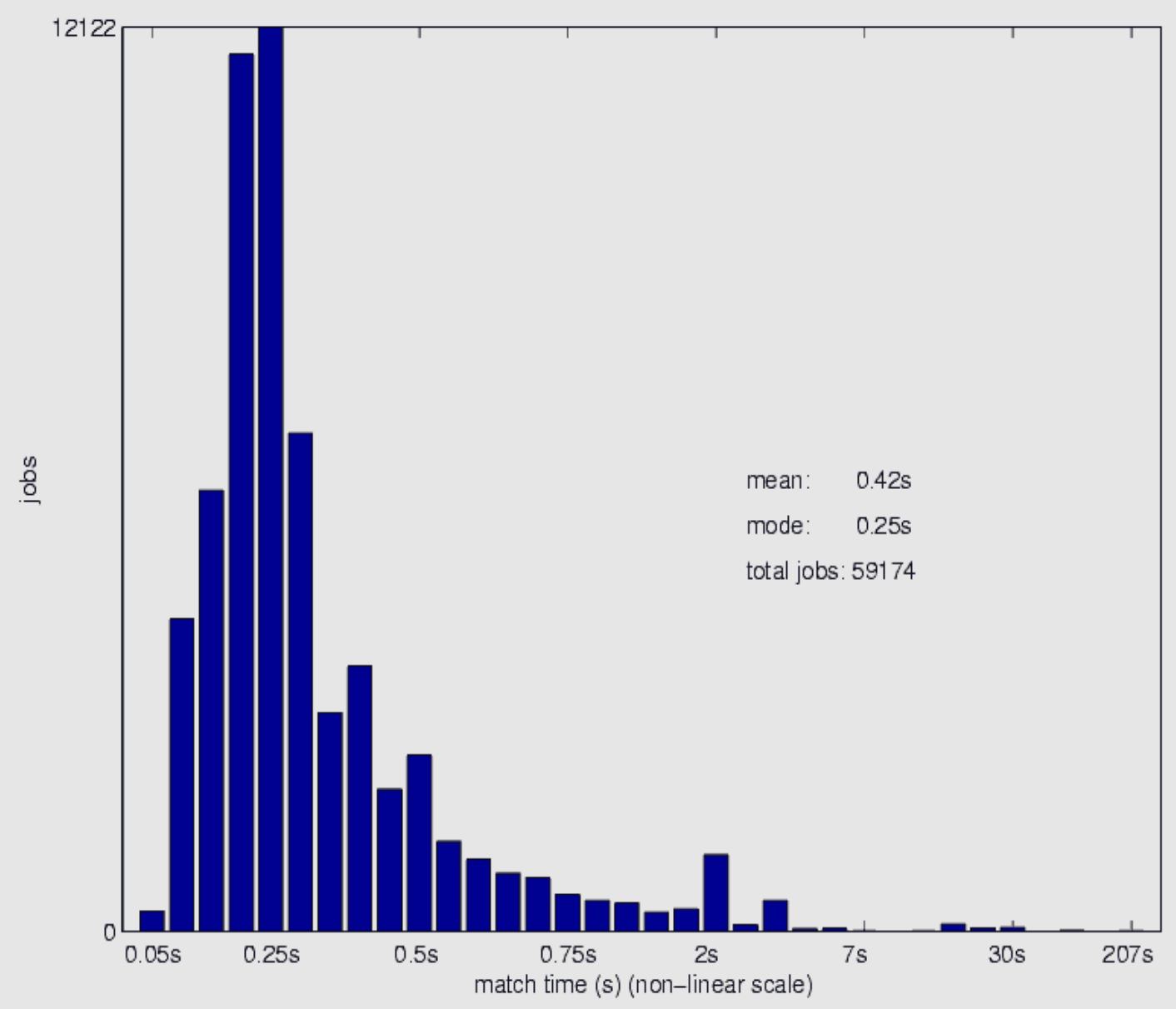


Lots of Agents, Clients and Services

* Changing location

* Restricted network access

* Need for reliable two-way communication

J) Jabber softwarefoundation

* Idea: Use asynchronous, buffered, reliable messaging framework - Jabber/XMPP IM 
* "Chat Rooms" provide ad hoc broadcast messaging hubs, and dynamic list of "active" jobs, services, agents, clients.

- Information/Query mechanism can be used to expose RPC API

* Presence can be used for component status

* Connection based:

> "tunnel" back to component, even if on NAT and/or behind firewall

$>$ Authenticate once

* Humans can interact with components using standard IM client - just open a chat session! 


\section{Experiences}


* Initial plan for DIRAC v2 was to implement all services with OGSI

> ideally pyOGSI or pyGridWare (stay 100\% Python)

$>$... but GT3 and maybe Jython would do in a pinch

* Conceptually, OGSI was excellent

* In practice, it was too complicated

- And GT3 was impossible to work with

$>$ Insufficient documentation

$>$ Buggy implementation

$>$ Performance was terrible

$>$ Development was arduous 
1. Robust libraries

2. Good documentation

tutorials, APIs, installation, developers guide, FAQ

3. Conceptually simple

4. Ease of installation

5. Ease of development

6. Smooth integration with existing tools

Tomcat, Axis, Globus

7. Performance

8. Scalability

9. Portability

10. Lightweight clients

11. Operation in unprivileged user space
Of course we expect it to work with:

$>$ expert administrator, "root" access

$>2$ gigs free hard drive space

$>512$ megs of RAM

$>100 \%$ "default" install

$>10-100$ services deployed

$>$ No firewall

$>$ Access only from other systems and users who are similarly equiped
But will it work with:

$>1000$ services on one machine

$>5000$ connections to one service

$>10,000$ grid jobs running at once

$>$ jobs interacting with 100 services

$>$ services distributed across 20 machines at 5 sites

$>20,000$ users, many novice 
* Transition from "classic" computing centres to "grid" computing was achieved

> Started 100\% classic, 0\% LCG (600-1200 jobs)

$>$ Soon moved to $80 \%$ classic, 20\% LCG (1000-1500 jobs)

$>$ Finished at 20\% classic, 80\% LCG (2500-3500 jobs)

* Initial efforts to utilise LCG were plagued by endless problems:

$>$ Jobs aborted mysteriously

$>$ Jobs disappearing

$>$ Queue times not as reported

$>$ Difficult to submit large numbers of jobs 


\section{EridPP}

* LCG were very supportive

$>$ Assigned two support contacts

> Provided 3 dedicated LHCb Resource Brokers

$>$ Arranged weekly phone conferences

* But it was still difficult to run 3000+ jobs a day on LCG

$>$ Resource Broker couldn't cope

$>$ Commands not designed for large numbers of jobs

$>$ Difficult to diagnose problems

* Heroic efforts by Ricardo Graciani (LHCb member from Barcelona) and collaboration with LCG team got new RB in place and running $4000+$ jobs at $>95 \%$ success. 

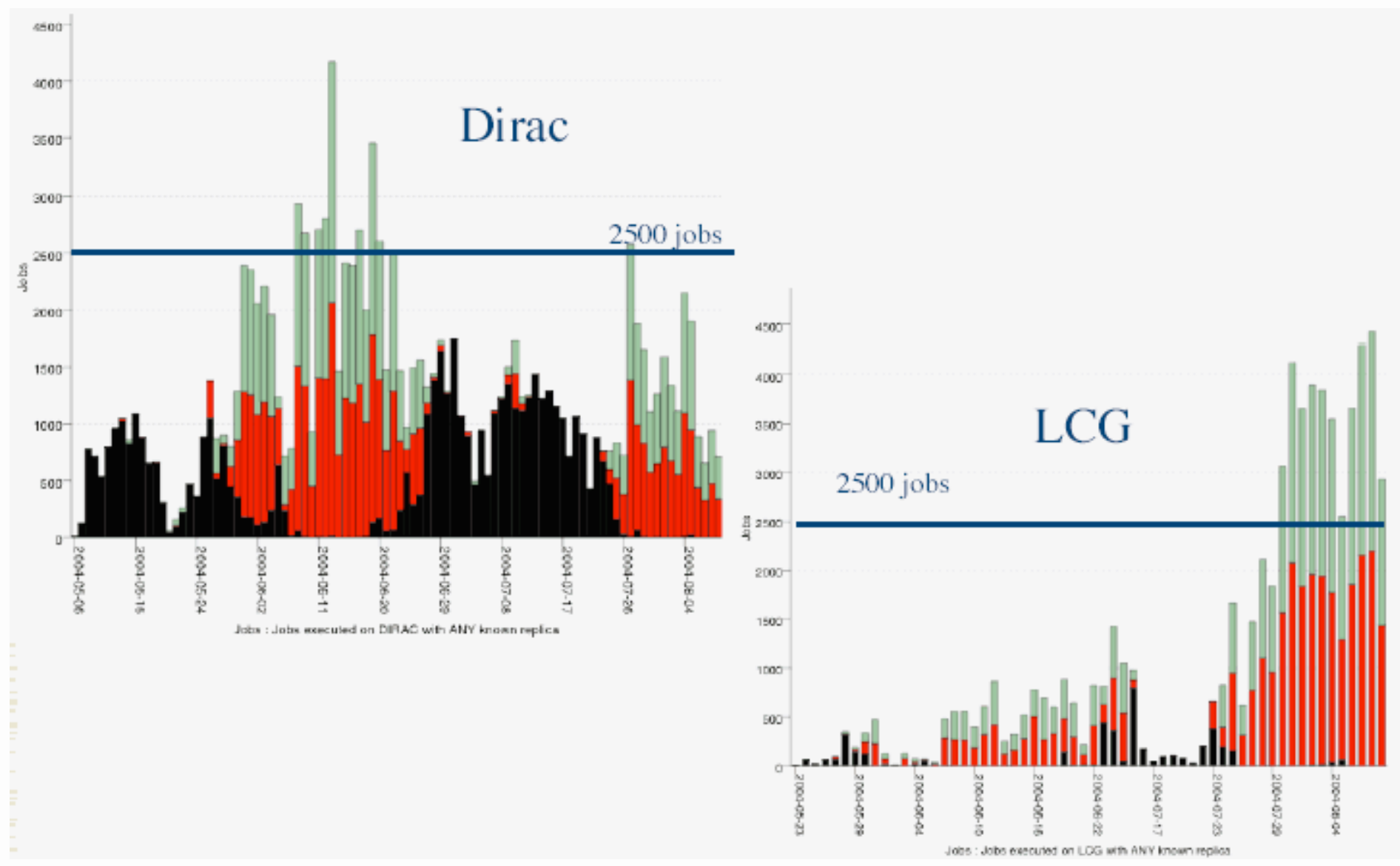
* Queue time normalisation

$>$ Hyper Threading

$>$ Overloading

* Job scratch space

$>$ Not enough

* Output files erased

$>$ Made debugging impossible

* Security certificates

$>$ RB used wrong ones
* Working with large numbers of jobs

$>$ Almost impossible

- Major problems with RB

$>$ Largely resolved now

* Lack of API documentation 
* On the grid, if something

can go wrong, it will:

$>$ Network failures

$>$ Drive failures

$>$ Systems hacked

$>$ Power outage

$>$ Bugs in code

$>$ Flaky memory (parity errors)

$>$ Time outs

> Overloaded machine/service

$>$ Simultaneous operations (mutex, thread safety) 
* Everything must be fault tolerant, because faults are guaranteed to happen

$>$ Retries

$>$ Duplication

$>$ Fail-over

$>$ Caching

$>$ Watchdogs
* runit package was incredible

$>$ Watchdog

$>$ Auto-restart

$>$ Daemons

$>$ Auto-logging with timestamps

$>$ Setuid

$>$ Log rotation

$>$ Dependency mgmt

$>$ Sending signals 


\section{Human Factors for a Successful Grid Project}


* Project management was key to success of DIRAC development and DC04 grid computing

* Three interest groups

$>$ Core DIRAC developers

$>$ Physicists and managers for simulation

$>$ Computing site representatives 
* Weekly phone meetings

$>$ Between developers and simulation managers

$>$ Between site reps and simulation managers

* Two mailing lists

$>$ One for developers and planning

$>$ One for discussing ongoing simulations

* Quarterly "LHCb Software Weeks" at CERN 
* Use of CVS from outset (and WebCVS)

* Tied in to CERN Savannah System

$>$ Bug Tracking

$>$ Task Tracking

$>$ Support Requests

$>$ Excellent Software Project Mgmt Tool!

* Project Wiki for workbook and notes

$>$ Now using GridSite 


\section{EridPP}

UK Computing for Particle Physics

\section{Savannah Bug Tracker}

\begin{tabular}{|c|c|c|c|c|}
\hline Item ID & Summary & Submitted On & Assigned To & Submitted By \\
\hline 4218 & Option to provide environment variable on command line & 2004-Jul-23 16:03 & None & stokes \\
\hline 4159 & Upgrades mean sites are unavailable for long periods of time & 2004-Jul-19 08:27 & None & stokes \\
\hline 4084 & Difficult to access LCG tools & 2004-Jul-08 15:52 & None & stokes \\
\hline 4053 & Mystery job abortion & 2004-Jul-07 10:03 & None & stokes \\
\hline 3926 & Log file format is not easily parseable & 2004-Jun-28 14:43 & None & stokes \\
\hline 3925 & LCG lacks tools for users to efficiently managing large numbers of jobs & 2004-Jun-28 14:37 & None & stokes \\
\hline 3924 & RB Ixn1176 reinstalled from scratch & 2004-Jun-28 14:24 & None & stokes \\
\hline 3909 & Cannot specify maximum run time for jobs & 2004-Jun-25 18:01 & None & stokes \\
\hline 3904 & LCG queue times need to offer normalised value & 2004-Jun-25 15:09 & None & stokes \\
\hline 3902 & Job working directories don't have enough space or are NFS mounted & 2004-Jun-25 13.58 & None & stokes \\
\hline 3873 & EstimatedResponseTime values for site ranking are completely bogus & 2004-Jun-24 11.51 & None & stokes \\
\hline 3845 & LCG sites going down with little notice & 2004-Jun-22 13:44 & None & stokes \\
\hline 3838 & Jobs aborted with message "canceled by user", but user didn't cancel them & 2004-Jun-22 09:54 & None & stokes \\
\hline 3831 & Submitting about 1000 jobs a day is too much for the RB & 2004-Jun-21 12:57 & None & stokes \\
\hline 3822 & Fuzzy selection to avoid all jobs going to same site is broken & 2004-Jun-19 07:50 & None & stokes \\
\hline 3789 & std out and std err not returned on aborted/canceled jobs & 2004-Jun-17 12:45 & None & stokes \\
\hline 3785 & RB restarting jobs in progress & 2004-Jun-17 10:54 & None & stokes \\
\hline 3764 & Proxy expiry & 2004-Jun-16 07:52 & None & stokes \\
\hline
\end{tabular}




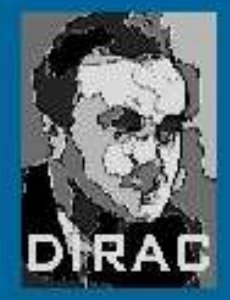

Savannah

Bugs

Support

cys

Downloads

Documentation 8. Publications

Mailing Lists

FAQ

Twiki

Team

Supporters

LHCb DCO4

Gridsite Guide

$\mathrm{LHCb}$ Thas

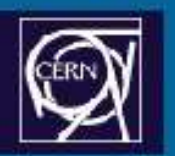

\section{LHCb Data Challenge 2004}

The Data Challenge has four goals:

1. Produce $60 \mathrm{~TB}$ of Monte Carlo simulation data for later analysis

2. Excercise the LHCb software tools and computing infrastructure

3. Validate the useability of LCG computing resources

4. Demonstrate the ability to perform distributed analysis

There are 20 sites explicitly participating in the Data Challenge, with a further 40 sites participating indirectly through LCG. Participants

\section{Monitoring and Accounting}

\section{Production System:}

Monitoring: http://fpegaes1.usc.es/dmon/DC04/joblist.html

Accounting: http://hcb.ecm.ub.es/DCo4/Accounting/

Backup Monitoring: http://hcb02.usc.cesga.es/dmon/DC04/joblist. html

\section{Test System:}

Monitoring: http:/fpegaes1.usc.es/dmon/DCo4test/joblist.html

Accounting: http://hcb,ecm,ub.es/DCo4test/Accounting/

DC04 Summary Tables; http://lbnts3.cern.ch:8110/BkkSummary?-summary=index.htm

Last modified Tue 31 August 2004, Wiew page history

You are $/ \mathrm{C}=\mathrm{UK} / \mathrm{O}=\mathrm{e}$ science $/ \mathrm{OU}=0 \times$ ford $/ \mathrm{L}=0 \mathrm{e}$ SClCN=Ian Stokes-rees

Edit page. Manage directory. Switch to HTTP. Website Help. Print View, Built with Gridsite 1,0.4 


\section{EridPP \\ UK Computing for Particle Physics \\ Web Based Job Monitoring}

\section{Details}

Production ID:

All $\quad=$

Site:

\begin{tabular}{|l|l|}
\hline All & Al \\
\hline ANY & \\
CERN & \\
CERN.tsar-agent.ch \\
DIRAC.atsareg-cern.ch
\end{tabular}
Job Status:

All $\quad \longrightarrow$

App Status:

All

Boole execution, step 4 Boole, step 4 done

Brunel execution, step 3

Owner:

$\mathrm{Al}$

Max results:

Job Ids:

Jobs updated after: $25 / 08 / 2004$

Submit Reset

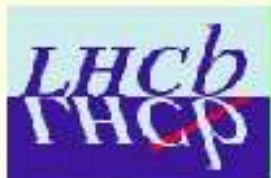

LHCb DC'04 Monitoring (test)

Showing 40 out of 1271 jobs. Pages: 1234567891011121314151617181920212223242526272829303132

\begin{tabular}{|c|c|c|c|c|c|c|}
\hline JobId & JobStatus & AppState & Site & JobName & Last Update & Owner \\
\hline$\underline{8854}$ & waiting & Unknown & LCG.TAU il & 00005615 _00000033 & $\begin{array}{c}2004-09-01 \\
11: 53: 28\end{array}$ & Thebprod \\
\hline$\underline{8855}$ & waiting & Unknown & LCG.TAU il & 00005615 _00000034 & $\begin{array}{c}2004-09-01 \\
11: 53: 29\end{array}$ & Thebprod \\
\hline$\underline{8856}$ & waiting & Unknown & LCG.TAU.il & 00005615 _00000035 & $\begin{array}{c}2004-09-01 \\
11: 53: 31\end{array}$ & Thebprod \\
\hline$\underline{8857}$ & waiting & Unknown & LCG.TAU il & 00005615 _00000036 & $\begin{array}{c}2004-09-01 \\
11: 53: 33\end{array}$ & lhcbprod \\
\hline$\underline{8858}$ & waiting & Unknown & LCG.TAU il & 00005615 _00000037 & $\begin{array}{c}2004-09-01 \\
11: 53: 35\end{array}$ & Thebprod \\
\hline$\underline{8859}$ & waiting & Unknown & LCG.TAU il & 00005615 _00000038 & $\begin{array}{c}2004-09-01 \\
11: 53: 37\end{array}$ & thebprod \\
\hline$\underline{8860}$ & waiting & Unknown & LCG.TAU.il & 00005615 _00000039 & $\begin{array}{c}2004-09-01 \\
11.53: 38\end{array}$ & Thebprod \\
\hline 8861 & waiting & Unknown & LCG.TAU.il & 00005615 _00000040 & $\begin{array}{c}2004-09-01 \\
11: 53: 40\end{array}$ & lhcbprod \\
\hline
\end{tabular}


- Typical Job

$>2$ GB local storage

> 300-600 MB transferred at end

$>15-24$ hours execution
- 58 TB of data produced

* 175M events

* 50+ sites

- 400 CPU years

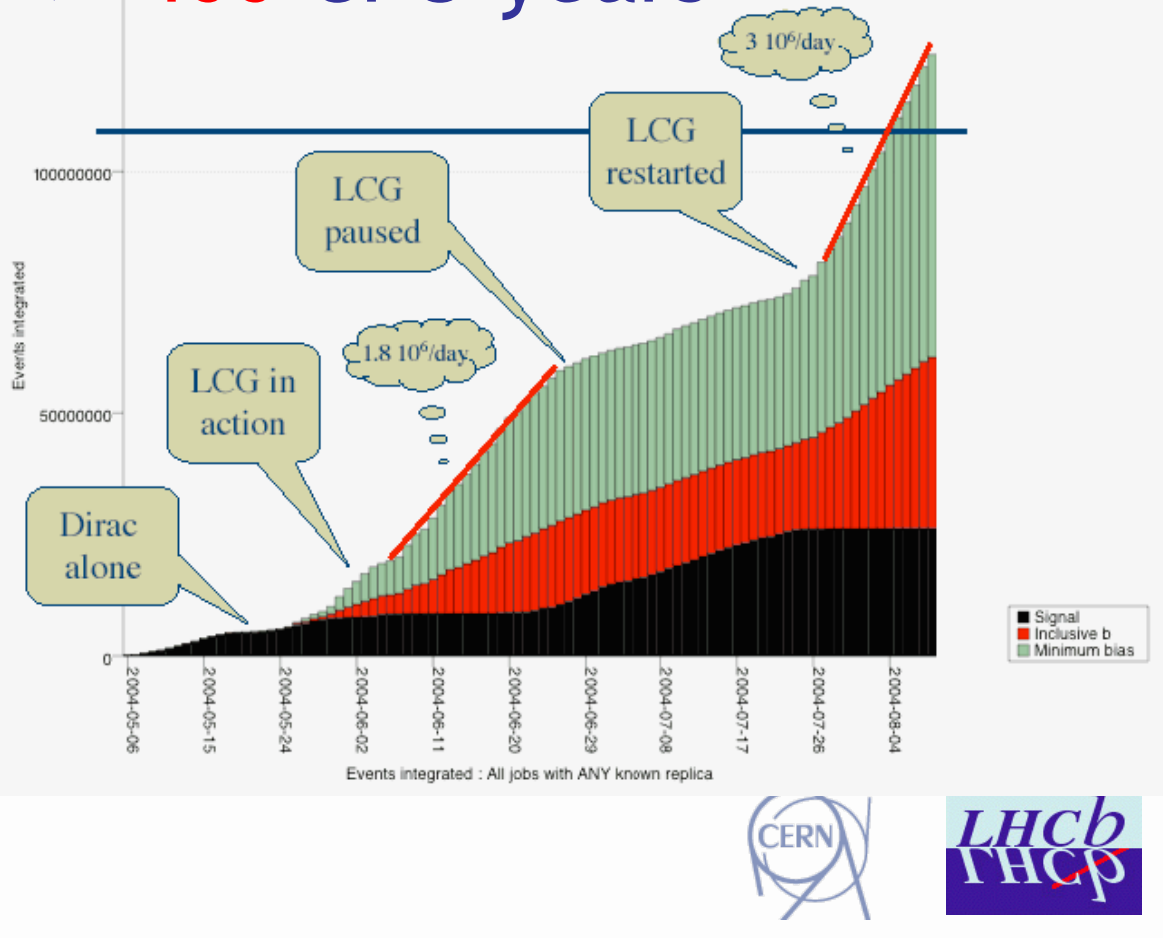


- Increased integration with LCG

* Investigation of gLite

* May look at WSRF and GT4 (no promises)

* Expose services via Apache, mod_python, and mod_gridsite

* (much) better security mechanisms

* Explore Instant Messaging opportunities 
For further information on DIRAC and LHCb:

GridSite:

http://dirac.cern.ch

email:

Ian Stokes-Rees

i.stokes-rees1@physics.ox.ac.uk

... or talk to me at the break
DIRAC and the results from DC04 are the result of many peoples efforts and the support of numerous participating institutions:

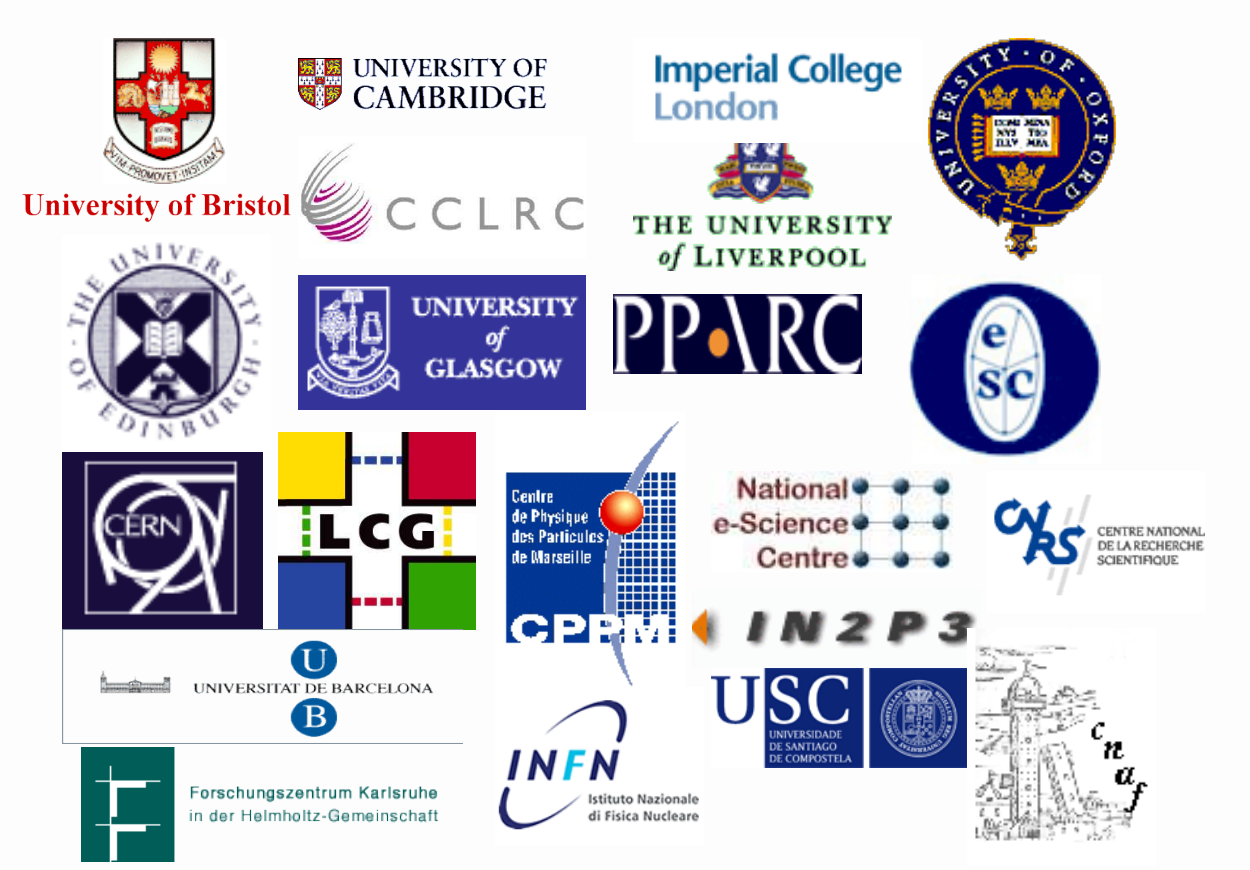

